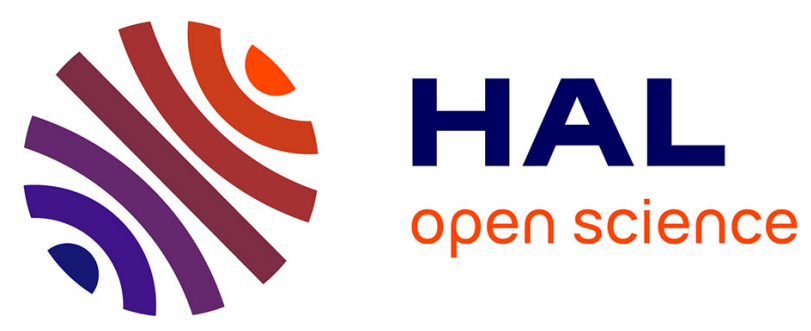

\title{
One Mesh To Rule Them All: Registration-Based Personalized Cardiac Flow Simulations
}

Alexandre A This, Ludovic Boilevin-Kayl, Hernán G Morales, Odile Bonnefous, Pascal A Allain, Miguel A Fernández, Jean-Frédéric Gerbeau

\section{To cite this version:}

Alexandre A This, Ludovic Boilevin-Kayl, Hernán G Morales, Odile Bonnefous, Pascal A Allain, et al.. One Mesh To Rule Them All: Registration-Based Personalized Cardiac Flow Simulations. FIMH 2017 - 9th international conference on Functional Imaging and Modeling of the Heart., Pop M and Wright G, Jun 2017, Toronto, Canada. hal-01512309

\section{HAL Id: hal-01512309 \\ https://hal.inria.fr/hal-01512309}

Submitted on 22 Apr 2017

HAL is a multi-disciplinary open access archive for the deposit and dissemination of scientific research documents, whether they are published or not. The documents may come from teaching and research institutions in France or abroad, or from public or private research centers.
L'archive ouverte pluridisciplinaire HAL, est destinée au dépôt et à la diffusion de documents scientifiques de niveau recherche, publiés ou non, émanant des établissements d'enseignement et de recherche français ou étrangers, des laboratoires publics ou privés. 


\title{
One Mesh To Rule Them All: Registration-Based Personalized Cardiac Flow Simulations
}

\author{
Alexandre This ${ }^{1,2,3(\bowtie)}$, Ludovic Boilevin-Kayl ${ }^{2,3}$, Hernán G. Morales ${ }^{1}$, \\ Odile Bonnefous $^{1}$, Pascal Allain ${ }^{1}$, Miguel A. Fernández ${ }^{2,3}$ \\ and Jean-Frédéric Gerbeau ${ }^{2,3}$ \\ 1 Medisys, Philips Research, France \\ 2 Inria Paris, 75012 Paris, France \\ 3 Sorbonne Universités, UPMC Univ Paris 6, UMR 7598 LJLL, 75005 Paris, France \\ $\bowtie$ alexandre.this@philips.com
}

\begin{abstract}
The simulation of cardiac blood flow using patient-specific geometries can help for the diagnosis and treatment of cardiac diseases. Current patient-specific cardiac flow simulations requires a significant amount of human expertise and time to pre-process image data and obtain a case ready for simulations. A new procedure is proposed to alleviate this pre-processing by registering a unique generic mesh on patient-specific cardiac segmentations and transferring appropriately the spatiotemporal dynamics of the ventricle. The method is applied on real patient data acquired from 3D ultrasound imaging. Both a healthy and a pathological conditions are simulated. The resulting simulations exhibited physiological flow behavior in cardiac cavities. The experiments confirm a significant reduction in pre-processing work.
\end{abstract}

Keywords: Patient-Specific, Numerical Simulation, Registration, Mitral Regurgitation

\section{Introduction}

In order to reduce the deadly impact of cardiovascular diseases, efforts are made to improve diagnosis and treatments of patients. Numerical simulations of blood flow within the cardiac cavities provide a better understanding of the intraventricular hemodynamics and hence could be used to improve the diagnosis or predict the outcome of treatments [1].

The current trend for patient-specific computational fluid dynamics (CFD) is to rely on image data to personalize the models [2,3]. Several sequential steps are usually required. The segmentation of images is nowadays almost automated thanks to robust image processing algorithms. The resulting segmented surfaces, however, often requires specific manual edition as well as a volumic meshing step before being ready for numerical simulations. Those manual edition might involve cleaning the mesh, refining regions of interest, including valves, labeling 


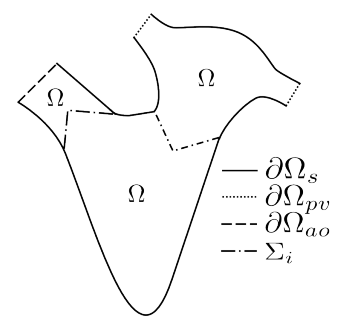

A)

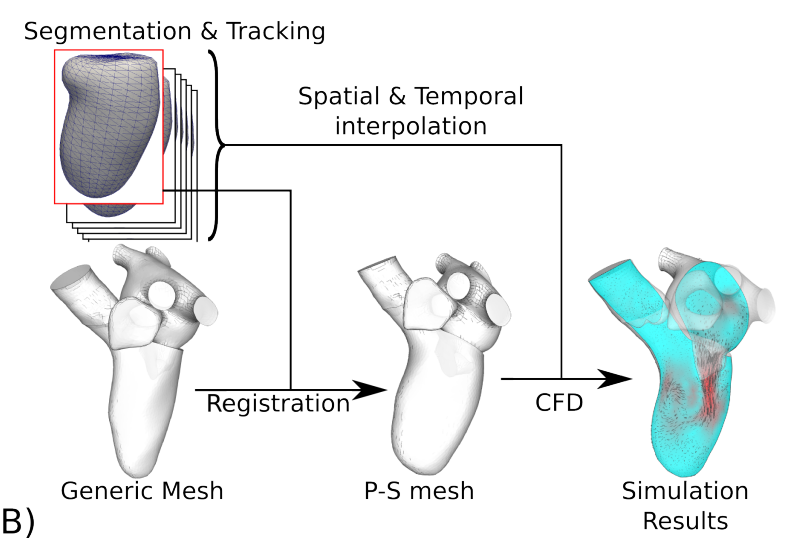

Fig. 1. A) Problem domain and B) pipeline schematic

the mesh surfaces for boundary condition and extruding inlets and outlets to reduce the effect of boundary conditions. Additionally, in the context of cardiac flow simulations, those pre-processing steps also involve the extraction and manipulation of the dynamics of the segmented surface in order to provide appropriate displacement boundary conditions. It has been reported in a recent review of patient-specific cardiac flow simulations that around "20-50 hours of human effort are needed for these pre-processing steps" [1] and similar claims have been made by others [13].

A new procedure is presented in order to reduce pre-processing time. First, real-time 3D echocardiography (RT3DE) data acquired on a patient were used to register a pre-processed generic mesh. Mesh morphing method have been used previously in bone mechanics $[11,12]$. Here we propose to use a similar methodology to obtain a patient-specific geometry from US image segmentations. The spatiotemporal data from the patient describing the ventricle dynamics are then transferred on the registered mesh to perform a patient-specific CFD simulation. Modifying a simplified valve model, the proposed framework allows to deliver physiological pressures during the isovolumic phases and this model has further been modified to allow the modeling of mitral regurgitation (MR), a pathology referring to a defect of the mitral complex that causes a backflow of blood into the atrium during ventricular systole, without any additional modifications of the generic mesh.

\section{Methods}

\subsection{Mathematical Modeling}

The generic computational domain (see Figure 1) includes the left ventricle (LV), the left atrium (LA) and a part of the ascending aorta. Both the aortic and the mitral valves were also included in the domain. Blood flow was described using 
the Navier-Stokes equations for a Newtonian incompressible fluid. In order to account for the motion of the computational domain, the equations were considered in their Arbitrary Lagrangian Eulerian (ALE) formulation. The aortic and mitral valves were modeled using a simplified resistive immersed surfaces (RIS) model [4], for which a dissipative term is added in the system. This dissipative term can be interpreted as a penalization of the fluid velocity for which the penalty parameter depends on the open or closed state of the valve. In this work, two extensions of the RIS model are considered. First, to model valve regurgitation, a space and time varying resistive term $R(\boldsymbol{x}, t)$ is considered allowing a finer control on which immersed elements are affected. The second extension concerns the isovolumic phases of the heart cycle. Generally speaking, when a fluid is enclosed in a cavity under Dirichlet boundary conditions, the pressure is only determined up to a constant. Numerically, the RIS model overcomes this issue by imposing a Dirichlet boundary condition via penalization. Nevertheless, this pressure is not correctly determined. The RIS model has been enhanced so that the intraventricular pressure is corrected, fitting a given pressure function $\overline{P_{v}}(t)$ inside the ventricle. This additional pressure data can be obtained by several means (e.g: measurements or numerical simulations). Defining + as the distal part (the outside of the ventricle), $\boldsymbol{n}_{\Sigma}$ as the outer-pointing normal vector of the surface $\Sigma$ and $[[\cdot]]_{\Sigma}$ as the jump operator across the surface $\Sigma$, the interface condition on the valves are:

$$
\left[\left[\boldsymbol{\sigma}(\boldsymbol{u}, p) \cdot \boldsymbol{n}_{\Sigma}\right]\right]_{\Sigma}=-R_{\Sigma}(\boldsymbol{x}, t)(\boldsymbol{u}-\boldsymbol{w})+\left(P_{\Sigma}^{+}-\overline{P_{v}}(t)\right) \cdot \boldsymbol{n}_{\Sigma}
$$

The full problem is therefore written as :

$$
\left\{\begin{aligned}
& \rho\left(\left.\frac{\partial \boldsymbol{u}}{\partial t}\right|_{\mathcal{A}}+(\boldsymbol{u}-\boldsymbol{w}) \cdot \nabla \boldsymbol{u}\right)-\nabla \cdot \boldsymbol{\sigma}(\boldsymbol{u}, p) \\
&=\sum_{i=\{o, c\}}\left[-R_{\Sigma_{i}}(\boldsymbol{x}, t)(\boldsymbol{u}-\boldsymbol{w})+\left(P_{\Sigma_{i}}^{+}-\overline{P_{v}}(t)\right) \cdot \boldsymbol{n}_{\Sigma_{i}}\right] \delta_{\Sigma_{i}}, \Omega(t) \\
& \nabla \cdot \boldsymbol{u}=0,, \Omega(t) \\
& \boldsymbol{u}=\boldsymbol{u}_{s}, \partial \Omega_{s} \\
& \boldsymbol{\sigma}(\boldsymbol{u}, p) \cdot \boldsymbol{n}=-P_{p v} \boldsymbol{n}, \partial \Omega_{p v} \\
& \boldsymbol{\sigma}(\boldsymbol{u}, p) \cdot \boldsymbol{n}=-P_{a o} \boldsymbol{n}, \partial \Omega_{a o}
\end{aligned}\right.
$$

Here, $\rho$ is the fluid density, $\boldsymbol{u}$ is the fluid velocity, $\boldsymbol{w}$ is the domain velocity, $\boldsymbol{\sigma}(\boldsymbol{u}, p)=-p \boldsymbol{I}+2 \mu \boldsymbol{\epsilon}(\boldsymbol{u})$ is the fluid Cauchy stress tensor where $p$ is the fluid pressure, $\mu$ is the fluid dynamic viscosity and $\boldsymbol{\epsilon}(\boldsymbol{u})=\frac{1}{2}\left(\nabla \boldsymbol{u}+\nabla \boldsymbol{u}^{T}\right)$ is the strain rate tensor. $\boldsymbol{u}_{s}$ is the velocity satisfying a no-slip boundary condition on the solid surfaces, $P_{p v}$ is the pulmonary vein pressure and $P_{a o}$ is the aortic pressure calculated from a coupled 0D Windkessel RCR model. This system was discretized using P1/P1 stabilized finite elements. The surface displacements are extended within the left heart cavities using an appropriate incremental elastic operator whose Lamé parameters are updated element-wise to stiffen the smallest elements $([15,14])$. This approach allows to efficiently handle large deformations without compromising the validity of the mesh. 


\subsection{Generic Mesh Registration}

The presented work aims to bypass the tedious patient-specific mesh and data pre-processing. The main idea, described in this section, is to design a unique generic mesh and to combine it with image data to automatically generate patient-specific geometries and boundary conditions.

The computational domain was generated from the Zygote 3D Human Heart Model $^{4}$ obtained from highly resolved MRI and CT images. The computational domain is restricted to the LV, LA and a portion of the ascending aorta (Figure 1). While the original model contained segmentation of the aortic and mitral valve in their closed state, the open mitral valve configuration was not available. Therefore, it was modeled based on anatomical data $[5,6]$ using the CAD software Salome $e^{5}$. Particular attention has been paid to proper design of valvular leaflets substructure as recommended in [6]. The model was then post-processed using the software 3-Matic and a tetrahedral mesh was generated using GHS3D. This computational domain is referred as the generic mesh, which can be used in combination with any patient surface segmentation.

Using the software Qlab - 3DQAdvanced Plugin (Philips, Andover, MA), segmentation and tracking of the left ventricle is automatically performed on RT3DE images. A sequence of surfaces depicting the left ventricle is obtained with a time discretization of $25 \mathrm{~Hz}$. By interpolating the position of the vertices using cubic splines, a time discretization fitting the required simulation time steps is recovered. $D_{t_{n} \rightarrow t_{n+1}}^{s}$ denotes the displacement field of the vertices of the segmented mesh $s$ at time $t_{n}$ to the vertices at time $t_{n+1}$.

In order to adapt the generic mesh to the patient ventricular surface segmentations, a combination of rigid and non-rigid registration technique was used. A time reference $t_{0}$ was chosen as the starting point of the simulation. A coarse rigid registration step was first performed to align the generic mesh ventricle surface onto the ventricle segmentation. This alignment was achieved by matching the apexes, longitudinal and radial axis of both geometries. A non-rigid registration step was then performed. In this second step, it is convenient to preserve the generic mesh properties in order to remain suitable for CFD simulations. In that regard, the large deformation diffeomorphic metric mapping (LDDMM) framework implemented in the open-source software Deformetrica [7] was used to find a diffeomorphism that maps the generic LV surface to the segmented LV surface. This diffeomorphism is described as a displacement field $D_{g e n \rightarrow t_{0}}^{g}$ where the superscript $g$ denotes the definition on all the vertices of the generic ventricle mesh. The fields $D^{s}$ were then spatially interpolated onto the generic mesh using the normalized radial basis function (RBF) framework (2) with a gaussian RBF $\phi$ and a smoothing parameter $\lambda$.

$$
D_{t_{n} \rightarrow t_{n+1}}^{g}(\boldsymbol{x})=\frac{\sum_{i} D_{t_{n} \rightarrow t_{n+1}}^{s}\left(\boldsymbol{x}_{\boldsymbol{i}}\right) \phi\left(d_{i}, \lambda\right)}{\sum_{i} \phi\left(d_{i}, \lambda\right)} \text { with } d_{i}=\left\|\boldsymbol{x}-\boldsymbol{x}_{\boldsymbol{i}}\right\|
$$

\footnotetext{
${ }^{4}$ http://www.3dscience.com

${ }^{5}$ http://www. salome-platform.org
} 
Exploiting the ALE formulation, the displacement field $D_{g e n \rightarrow t_{0}}^{g}$ was used as a boundary condition of an initial mesh motion step allowing the full computational mesh to deform and fit the segmentation. The full problem described in Section 2.1 was then solved for all time steps using the displacement fields $D_{t_{n} \rightarrow t_{n+1}}^{g}$ as the ventricle surface boundary condition.

\section{Experiment}

We evaluated the proposed pipeline on one patient's dataset. Echocardiographic images were acquired using an IE33 ultrasound system (Philips, Andover, MA) equipped with a $1-5 \mathrm{MHz}$ transthoracic matrix array transducer (xMATRIX x51). RT3DE images were reconstructed as volumes over one cardiac cycle from acquisitions of sub-volumes over four cycles. The volumes were automatically segmented and tracked as previously described and used as sequence of 25 surface meshes. The cardiac cycles were replicated to allow the computation of several heart cycles. The generic mesh was composed of 178,348 elements for the proposed experiment. The registration process of Section 2.1 was applied to register the LV surface of the generic mesh onto the $\mathrm{LV}$ segmented surface. Time intervals of $1 \mathrm{~ms}$ were used for the time interpolation, and the spatial interpolation free parameter $\lambda$ was taken as the average Euclidean distance between vertices of the segmented mesh.

Once the appropriate displacement fields $D^{g}$ were computed, the fluid simulation problem described in Section 2.2 was solved using the finite element library FELiScE ${ }^{6}$. The blood parameters were $\mu=4$ Pas and $\rho=1060 \mathrm{~kg} / \mathrm{m}^{3}$. The simulation was run with a timestep of $1 \mathrm{~ms} . P_{p v}$ was set to $10 \mathrm{mmHg}$ while the 0D RCR model has the following parameter: $R_{\text {proximal }}=150, R_{\text {distal }}=2300$, $C=0.9, P_{\text {venous }}=0 \mathrm{mmHg}$ and $P_{\text {distal }, \mathrm{t}=0}=70 \mathrm{mmHg}$. Two heart cycles $(0.8$ seconds each) were simulated and the reported results were taken from the second one. Another simulation was performed with a circular hole of $0.5 \mathrm{~cm}^{2}$ in the mitral valve representing a severe MR [8].

\section{Results And Discussions}

The results of the registration steps are shown in Figure 2. The rigid registration aligns the two ventricles and the non-rigid registration process succeeds to deform the generic mesh onto the segmented mesh. It should be noted that the open-source software used allows for the tuning of an accuracy parameter. While this experiment uses a coarse kernel size, as recommended in the software documentation, the algorithm still provides good matching accuracy.

Time and space interpolation are then performed to produce the displacement fields $D^{g}$ at all times. The overlap between the image segmentation and the deformed generic mesh under the influence of the displacement fields $D^{g}$ is shown on Figure 3. This exhibits the ability of the proposed method to fit the generic

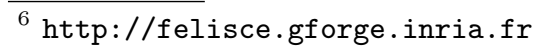



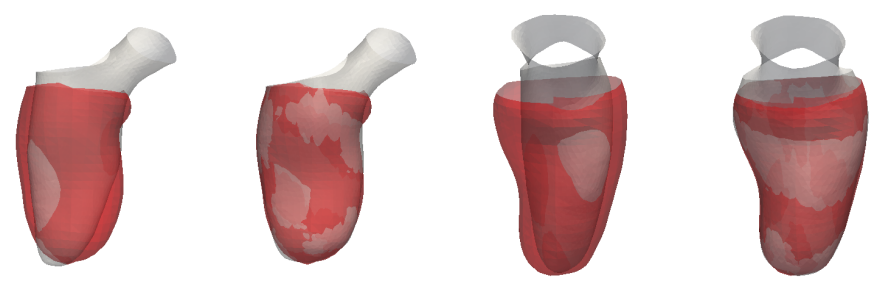

Fig. 2. Rigid and non-rigid step of the registration of the ventricle surfaces. The white surface is the generic mesh and the red surface is the output of the image segmentation

mesh onto the segmented surfaces. The complete pipeline was performed without human intervention in approximately $30 \mathrm{~min}$. Compared to state of the art methods, it brings several advantages: it decreases the amount of work needed between the acquisition of the images and the launch of the CFD simulation and removes the need of a meshing expert.
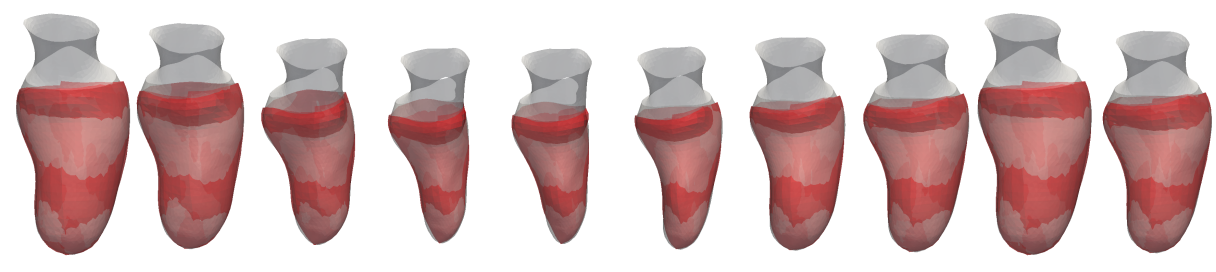

Fig. 3. Overlap of the segmented mesh and the deformed generic mesh over one cardiac cycle

The CFD simulations took 8 hours on a laptop using a Intel Core $i \%-4810 M Q$ cadenced at $2.8 \mathrm{GHz}$. The evolution of blood during the cardiac cycles is depicted in figure 4 . When the ventricular volume starts increasing, the mitral valve opens and blood enters the LV. The maximum blood velocity reaches approximately $100 \mathrm{~cm} / \mathrm{s}$ in the mitral valve area. One can notice the presence of a vortex that detaches from the tip of the mitral valve. Blood then slows down as the E-wave finishes. A second vortex appears inside the LV in the late diastole corresponding to the A-wave. Those complex flow structures are coherent with the ones reported in the literature $[2,9]$. In particular, the presence of the A-wave vortex is described in [2]. While the contraction of the atrium has not been simulated, the increase in LV volume recovered by the segmentation allows this behavior to appear in the presented simulation. As the LV contracts, the blood is then ejected at a speed of approximately $125 \mathrm{~cm} / \mathrm{s}$ through the aorta. The blood finally slows down before the next cardiac cycle. 

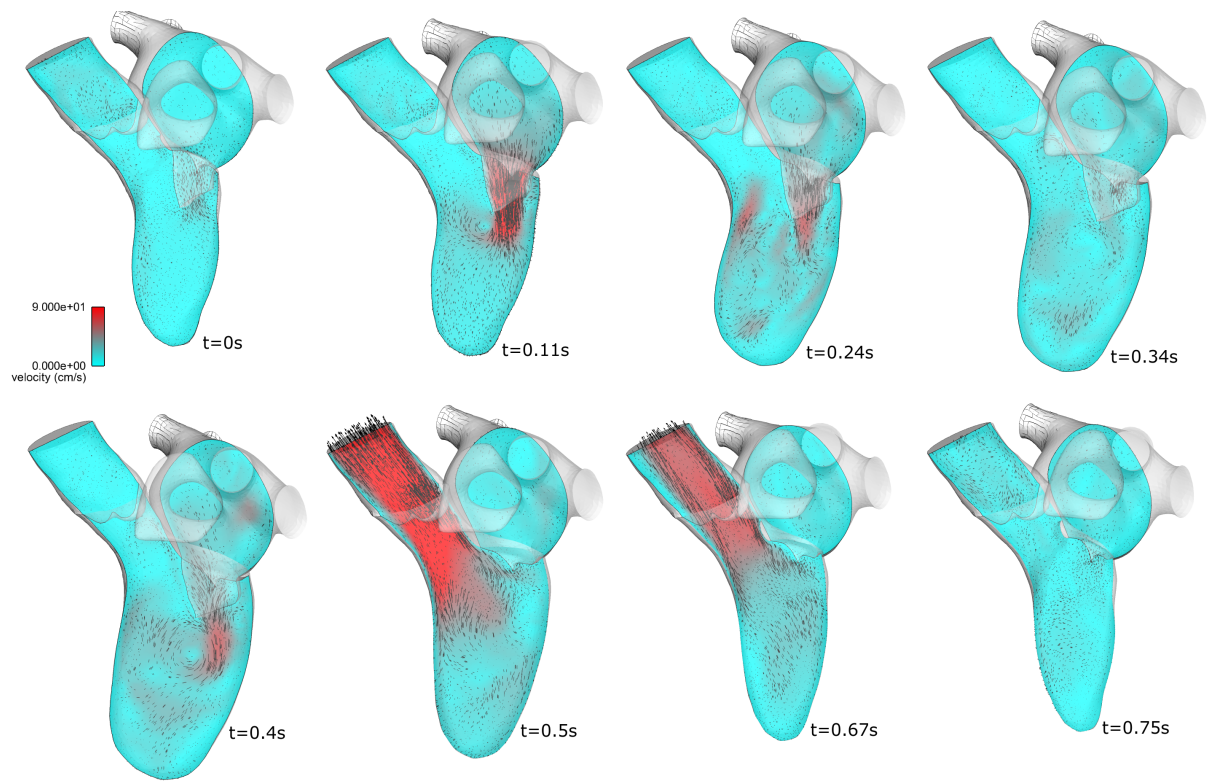

Fig. 4. Blood velocity vector field and magnitude on a cut plane

Figure 5 depicts the simulation including regurgitation. During the systole, a strong jet of blood comes back to the atrium through the mitral valve orifice. As reported in the litterature, the maximum velocity is obtained just downstream of the mitral valve regurgitation (vena contracta). The jet then hits the walls of the atrium. In the ventricular region, the blood velocity is forming flattened hemispherical isovelocity surfaces. This is coherent with the litterature on mitral quantification for such a hole geometry $[8,10]$.

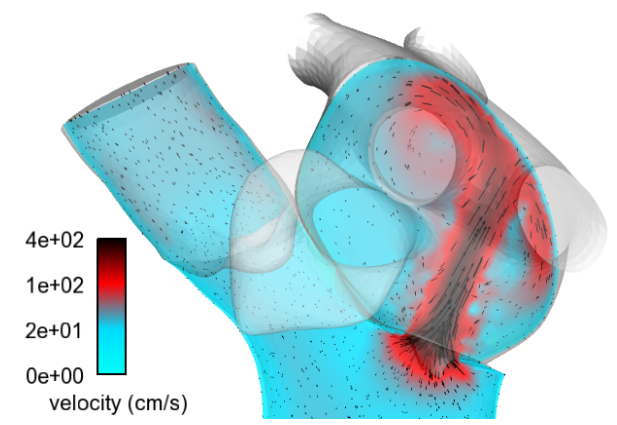

Fig. 5. Regurgitation case: flow convergence region and jet in the atrium 
One of the limitations of the proposed method is that it includes two free parameters ( $\lambda$ and the kernel size of the non-rigid algorithm). Fortunately, the impact of those parameter is intuitive (e.g. $\lambda$ for the RBF interpolation has a smoothing effect on the interpolation). Nevertheless, no quantitative analysis was performed in the presented work and further investigation should be performed. In this study, the $4 \mathrm{D}$ ventricular surface has been used to register a generic mesh that also includes a portion of the ascending aorta and the atrium. Therefore, one could argue that the rigid template of the atrium is arbitrary. While we did not have access to such data for the present article, a natural extension of the work would be to use $4 \mathrm{D}$ atrial segmentation within the same pipeline to improve the model. While in the current setting, the atrial geometry is indeed arbitrary, it is still a richer approach than imposing lumped atrial model at the mitral valve. In particular it avoids the imposition of unknown boundary conditions in a zone extremely close to the mitral valve, a region of interest.

Additionally, while the CFD simulations correctly captured the main hemodynamics features, comparisons should be done to assess the quality of the results. In particular, it is planned to add patient cases in the database and comparisons of hemodynamic quantities of interest using color Doppler images will allow to further validate the proposed framework. A more general limitation of patient-specific methods is concerning the loss of displacement information of the ventricle in the imaging stage. Indeed, the displacement in the normal direction of the surface is properly recovered but the tangential displacements are usually not (unless using specific imaging techniques such as tagged-MRI). The physiological twisting motion of the ventricle may hence be lost in the segmentation stage. Further work need to address this issue by quantifying the impact of the tangential ventricle velocity on the hemodynamics. Using a baseline simulation where the twisting motion is available, one could artificially remove the twisting motion and compare the main hemodynamic features of the two flow solutions. This problem could be solved by using anatomical landmarks visible in the images and using that information to constrain the segmentation process. Finally, to properly model patient-specific regurgitation, image data could be used to recover the patient-specific mitral valve regurgitant hole geometries.

\section{Conclusion}

A procedure was proposed to speed up the mesh pre-processing prior to CFD simulations of patient-specific cardiac blood flow. This procedure combines a rigid and non-rigid registration to morph a pre-computed unique generic mesh using patient-specific data. The ventricle dynamics is then transferred onto the registered mesh to prescribe appropriate boundary conditions. This pipeline was tested on RT3DE data and performed in around 30 min (improvement of around two order of magnitude compared to what is reported in the literature). To evaluate the pipeline, two CFD simulations were performed. The first simulation was done on a healthy patient, and in the second one, a hole in the mitral valve was added to obtain a severe MR. In both simulations, results showed flow 
patterns and hemodynamics quantities within the known cardiac physiology and blood velocities were coherent with the literature.

\section{Acknowledgements}

The authors gratefully acknowledge Mathieu De Craene, Èric Lluch and Hélène Langet from Philips Reasearch - Medisys for their support in acquiring patient data and their use, as well as for reviewing the presented manuscript. We would like to also acknowledge the help of INRIA - M3DISIM research team for sharing with us pressure curves generated from complex electro-mechanical simulations.

\section{References}

1. Mittal, R., Seo, J. H., Vedula, V., Choi, Y. J., Liu, H., Huang, H. H., George, R. T.: Computational modeling of cardiac hemodynamics: Current status and future outlook. Journal of Computational Physics, 305, 1065-1082. (2016)

2. Chnafa, C., Mendez, S., Nicoud, F.: Image-based large-eddy simulation in a realistic left heart. Computers And Fluids, 94, 173-187. (2014)

3. Bavo, A. M., Pouch, A. M., Degroote, J., Vierendeels, J., Gorman, J. H., Gorman, R. C., Segers, P.: Patient-specific CFD simulation of intraventricular haemodynamics based on 3D ultrasound imaging. BioMedical Engineering OnLine, 15(1), 107-122. (2016)

4. Astorino, M., Hamers, J., Shadden, S. C., Gerbeau, J.-F.: A robust and efficient valve model based on resistive immersed surfaces. International Journal for Numerical Methods in Biomedical Engineering, 28(9), 937-959. (2012)

5. Ranganathan, N., Lam, J. H., Wigle, E. D., Silver, M. D.: Morphology of the human mitral valve. II. The valve leaflets. Circulation, 41(3), 459-467. (1970)

6. Votta, E., Caiani, E., Veronesi, F., Soncini, M., Montevecchi, F. M., Redaelli, A.: Mitral valve finite-element modelling from ultrasound data: a pilot study for a new approach to understand mitral function and clinical scenarios. Philosophical Transactions of the Royal Society. Series A, Mathematical, Physical, and Engineering Sciences, 366(1879), 3411-3434. (2008)

7. Durrleman, S., Prastawa, M., Charon, N., Korenberg, J. R., Joshi, S., Gerig, G., Trouv, A.: Morphometry of anatomical shape complexes with dense deformations and sparse parameters. NeuroImage, 101(8), 35-49. (2014)

8. Lambert, A. S.: Proximal isovelocity surface area should be routinely measured in evaluating mitral regurgitation: A core review. Anesthesia and Analgesia, 105(4), 940-943. (2007)

9. Hong, G. R., Pedrizzetti, G., Tonti, G., Li, P., Wei, Z., Kim, J. K., Vannan, M. A.: Characterization and quantification of vortex flow in the human left ventricle by contrast echocardiography using vector particle image velocimetry. JACC. Cardiovascular Imaging, 1(6), 705-717. (2008)

10. This, A., Morales, H. G., Bonnefous, O.: Proximal isovelocity surface for different mitral valve hole geometries. In ECCOMAS Congress 2016 - Proceedings of the 7th European Congress on Computational Methods in Applied Sciences and Engineering 1, 155-163. (2016) 
11. Couteau, B., Payan, Y., and Lavallée, S.: The mesh-matching algorithm: an automatic 3D mesh generator for finite element structures - Journal of Biomechanics, 33(8),1005-1009 (2000)

12. Bucki, M., Lobos C., Payan, Y.: A fast and robust patient specific Finite Element mesh registration technique: Application to 60 clinical cases - Medical Image Analysis, 14(3),303-317 (2010)

13. Doost, S., Ghista, D., Su, B., Zhong, S., Morsi, Y. : Heart blood flow simulation: a perspective review.Biomedical engineering online, 15(1):101, (2016)

14. Stein, K., Tezduyar, T., and Benney, R. : Mesh Moving Techniques for FluidStructure Interactions With Large Displacements. Journal of Applied Mechanics, 70(1):58, (2003).

15. Landajuela, M., Vidrascu, M., Chapelle, D., Fernández, M. A. : Coupling schemes for the FSI forward prediction challenge: Comparative study and validation. International Journal for Numerical Methods in Biomedical Engineering, (2016). 